\title{
Publisher's Note: Ramsey spectroscopy, matter-wave interferometry, and the microwave-lensing frequency shift [Phys. Rev. A 90, 015601 (2014)]
}

Kurt Gibble

(Received 6 December 2017; published 29 December 2017)

DOI: 10.1103/PhysRevA.96.069910

This paper was published online on 14 July 2014 with errors in Eq. (4) and Eqs. (6) through (9). Equation (4) should read as

$$
\begin{aligned}
\delta P= & \left.\frac{1}{2}\left|\psi\left(\vec{r}, t_{d}\right)\langle f \mid \xi\rangle\right|_{\chi=\pi / 2}\right|^{2}-\left.\frac{1}{2}\left|\psi\left(\vec{r}, t_{d}\right)\langle f \mid \xi\rangle\right|_{\chi=-\pi / 2}\right|^{2} \\
= & \frac{1}{4}(-1)^{j_{e g}}\left\{\psi_{21^{\prime}}\left(\vec{r}, t_{d}\right) \psi_{22^{\prime}}\left(\vec{r}, t_{d}\right) \sin \left[\phi_{\beta}\left(\vec{r}_{2}\right)+\delta \Phi_{21^{\prime}}(\vec{r})-\delta \Phi_{22^{\prime}}(\vec{r})\right]-\psi_{11^{\prime}}\left(\vec{r}, t_{d}\right) \psi_{12^{\prime}}\left(\vec{r}, t_{d}\right) \sin \left[\phi_{\beta}\left(\vec{r}_{2}\right)\right.\right. \\
& \left.\left.+\delta \Phi_{11^{\prime}}(\vec{r})-\delta \Phi_{12^{\prime}}(\vec{r})\right]\right\}+\frac{1}{4}\left\{\psi_{11^{\prime}}\left(\vec{r}, t_{d}\right) \psi_{21^{\prime}}\left(\vec{r}, t_{d}\right) \sin \left[\phi_{\alpha}\left(\vec{r}_{1}\right)+\delta \Phi_{11^{\prime}}(\vec{r})-\delta \Phi_{21^{\prime}}(\vec{r})\right]\right. \\
& \left.-\psi_{12^{\prime}}\left(\vec{r}, t_{d}\right) \psi_{22^{\prime}}\left(\vec{r}, t_{d}\right) \sin \left[\phi_{\alpha}\left(\vec{r}_{1}\right)+\delta \Phi_{12^{\prime}}(\vec{r})-\delta \Phi_{22^{\prime}}(\vec{r})\right]\right\} .
\end{aligned}
$$

Equation (6) should read as

$$
\begin{aligned}
\delta P_{x}= & \frac{1}{2} \psi_{0 x}^{2}\left(x, t_{d}\right)\left((-1)^{j_{e g}}\left\{\frac{\delta_{\alpha x}}{w_{d x}}\left[1-2\left(\frac{x-x_{d}}{w_{d x}}\right)^{2}\right]-2 \varepsilon_{\alpha x}\left(\vec{r}_{1}\right) \frac{x-x_{d}}{w_{d x}^{2}}\right\} \sin \left[\phi_{\beta}\left(\vec{r}_{2}\right)\right]\right. \\
& \left.-(-1)^{l_{g e}}\left\{\frac{\delta_{\beta x}}{w_{d x}}\left[1-2\left(\frac{x-x_{d}}{w_{d x}}\right)^{2}\right]-2 \varepsilon_{\beta x}\left(\vec{r}_{2}\right) \frac{x-x_{d}}{w_{d x}^{2}}\right\} \sin \left[\phi_{\alpha}\left(\vec{r}_{1}\right)\right]\right) .
\end{aligned}
$$

Equation (7) should read as

$$
\begin{aligned}
\delta P_{x} & =\frac{1}{2}(-1)^{j_{e g}}\left[\psi_{2}^{2}\left(\vec{r}, t_{d}\right)-\psi_{1}^{2}\left(\vec{r}, t_{d}\right)\right] \sin \left[\phi_{\beta}\left(\vec{r}_{2}\right)\right] \\
& =\frac{(-1)^{j_{e g}}}{2} \psi_{0 x}^{2}\left(x, t_{d}\right)\left\{\frac{\delta_{\alpha x}}{w_{d x}}\left[1-2\left(\frac{x-x_{d}}{w_{d x}}\right)^{2}\right]-2 \varepsilon_{\alpha x}\left(\vec{r}_{1}\right) \frac{x-x_{d}}{w_{d x}^{2}}\right\} \sin \left[\phi_{\beta}\left(\vec{r}_{2}\right)\right] .
\end{aligned}
$$

Equation (8) should read as

$$
\begin{aligned}
\frac{\delta v}{v_{R}}= & \frac{(-1)^{l_{g e}} \phi_{\alpha}(0) \sin \left[\phi_{\beta}(0)\right] a\left(t_{2 L}-t_{1}\right)}{\sin \left[\phi_{\alpha}(0)\right] \sin \left[\phi_{\beta}(0)\right] k\left(t_{2}-t_{1}\right) \int_{r_{2 L 0}<a} \int_{r_{1 L}<a_{1 L}} \psi_{0}^{2}\left(\vec{r}_{2 L 0}, \vec{r}_{1 L}\right) d \vec{r}_{1 L} d \vec{r}_{2 L 0}} \\
& \times\left.\int_{0}^{2 \pi} \int_{r_{1 L}<a_{1 L}} \frac{r_{2 L 0}\left(t_{1}-t_{1 L}\right)+r_{1 L}\left(t_{2 L}-t_{1}\right) \cos \left(\phi_{2 L 0}\right)}{\left|\vec{r}\left(t_{1}\right)\right|\left(t_{2 L}-t_{1 L}\right)} J_{1}\left(k\left|\vec{r}\left(t_{1}\right)\right|\right) \psi_{0}^{2}\left(\vec{r}_{2 L 0}, \vec{r}_{1 L}\right)\right|_{r_{2 L 0}=a} d \vec{r}_{1 L} d \phi_{2 L 0}, \\
& \psi_{0}^{2}\left(\vec{r}_{2 L 0}, \vec{r}_{1 L}\right)=\exp \left[-\frac{r_{2 L 0}^{2} w_{1 L}^{2}+r_{1 L}^{2} w_{2 L}^{2}-2 r_{2 L 0} r_{1 L}\left(w_{0}^{2}+u^{2} t_{2 L} t_{1 L}\right) \cos \left(\phi_{2 L 0}\right)}{w_{0}^{2} u^{2}\left(t_{2 L}-t_{1 L}\right)^{2}}\right], \quad \vec{r}(t)=\frac{\vec{r}_{2 L 0}\left(t-t_{1 L}\right)+\vec{r}_{1 L}\left(t_{2 L}-t\right)}{t_{2 L}-t_{1 L}} .
\end{aligned}
$$

Equation (9) should read as

$$
\begin{aligned}
\frac{\delta v}{v_{R}}= & -\cos \left[\phi_{\alpha}(0)\right] \cos \left[\phi_{\beta}(0)\right] \frac{(-1)^{l_{g e}} \phi_{\beta}(0) \sin \left[\phi_{\alpha}(0)\right] a\left(t_{2 L}-t_{2}\right)}{\sin \left[\phi_{\alpha}(0)\right] \sin \left[\phi_{\beta}(0)\right] k\left(t_{2}-t_{1}\right) \int_{r_{2 L 0}<a} \int_{r_{1 L}<a_{1 L}} \psi_{0}^{2}\left(\vec{r}_{2 L 0}, \vec{r}_{1 L}\right) d \vec{r}_{1 L} d \vec{r}_{2 L 0}} \\
& \times\left.\int_{0}^{2 \pi} \int_{r_{1 L}<a_{1 L}} \frac{r_{2 L 0}\left(t_{2}-t_{1 L}\right)+r_{1 L}\left(t_{2 L}-t_{2}\right) \cos \left(\phi_{2 L 0}\right)}{\left|\vec{r}\left(t_{2}\right)\right|\left(t_{2 L}-t_{1 L}\right)} J_{1}\left(k\left|\vec{r}\left(t_{2}\right)\right|\right) \psi_{0}^{2}\left(\vec{r}_{2 L 0}, \vec{r}_{1 L}\right)\right|_{r_{2 L 0}=a} d \vec{r}_{1 L} d \phi_{2 L 0},
\end{aligned}
$$

The equations have been corrected as of 27 December 2017. The equations are incorrect in the printed version of the journal.

The author is grateful to S. R. Jeffers, T. P. Heavner, S. E. Barlow, and N. Ashby for bringing the typesetting errors to his attention. 